
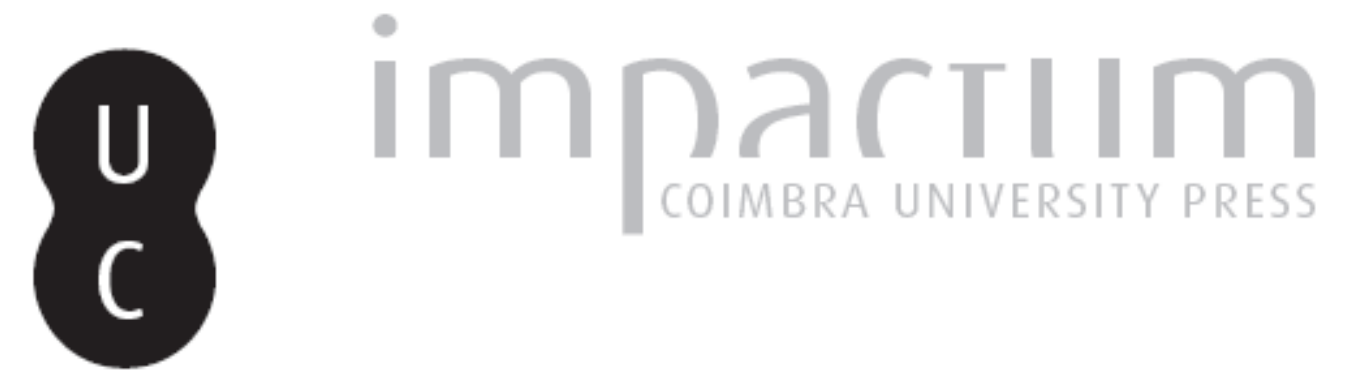

\title{
Friedrich Schleiermacher und das Platonbild des 19. und 20. Jahrhunderts
}

Autor(es): Szlezák, Thomas Alexander

Publicado por: Imprensa da Universidade de Coimbra

URL persistente:

URI:http://hdl.handle.net/10316.2/42272

DOI:

DOI:https://doi.org/10.14195/2183-4105_2_6

Accessed : $\quad$ 26-Apr-2023 14:14:42

A navegação consulta e descarregamento dos títulos inseridos nas Bibliotecas Digitais UC Digitalis, UC Pombalina e UC Impactum, pressupõem a aceitação plena e sem reservas dos Termos e Condições de Uso destas Bibliotecas Digitais, disponíveis em https://digitalis.uc.pt/pt-pt/termos.

Conforme exposto nos referidos Termos e Condições de Uso, o descarregamento de títulos de acesso restrito requer uma licença válida de autorização devendo o utilizador aceder ao(s) documento(s) a partir de um endereço de IP da instituição detentora da supramencionada licença.

Ao utilizador é apenas permitido o descarregamento para uso pessoal, pelo que o emprego do(s) título(s) descarregado(s) para outro fim, designadamente comercial, carece de autorização do respetivo autor ou editor da obra.

Na medida em que todas as obras da UC Digitalis se encontram protegidas pelo Código do Direito de Autor e Direitos Conexos e demais legislação aplicável, toda a cópia, parcial ou total, deste documento, nos casos em que é legalmente admitida, deverá conter ou fazer-se acompanhar por este aviso.

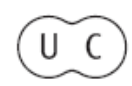




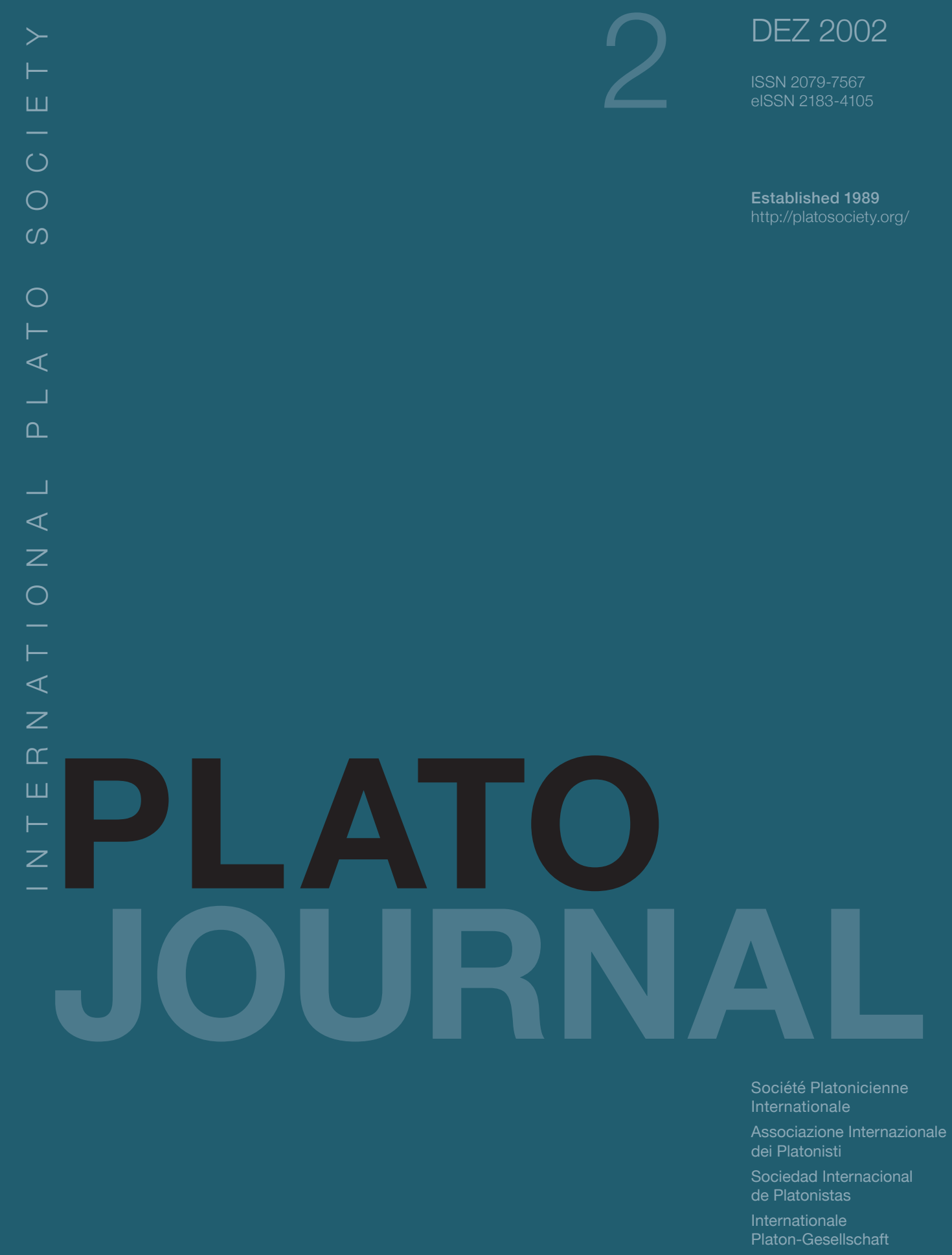




\title{
Friedrich Schleiermacher und das Platonbild des 19. und 20. Jahrhunderts
}

\author{
SZLEZÁK, Thomas Alexander, in 2. Plato \\ 2 (2002) , [En ligne] , January 2002
}

I

Schleiermachers berühmte Übersetzung der Dialoge Platons, die noch heute nachgedruckt und benützt wird, blieb bekanntlich unvollständig: es fehlen die Nomoi, der Timaios, das Kritias-Fragment und die Briefe. Doppelt unvollständig blieb dann auch seine Detailbeschäftigung mit platonischen Problemen: denn diese erfolgte in Form von Einleitungen und Erläuterungen zu den übersetzten Dialogen, unterblieb also für die genannten Werke, und die Erläuterungen waren von vornherein nicht als umfassende Kommentare gedacht, wozu Schleiermacher bei seinem Beruf als Prediger wohl auch nicht die Zeit gehabt hätte; sie blieben also selektiv. Schleiermacher war nicht Philologe (auch wenn er das Fach in Halle zwei Jahre studiert hatte), und er war von Beruf nicht Philosophiehistoriker.

Nimmt man diese Punkte zusammen, so gewinnt man rückblikkend den Eindruck, daß die Bedingungen, unter denen Schleiermachers Übersetzungswerk stand, nicht eben die optimalen waren, um dem Unternehmen einen nachhaltigen Einfluß zu sichern.

Doch gerade das ist eingetreten. Die Art, wie Schleiermacher an die Dialoge übersetzend, einleitend und erläuternd heranging, hat einen weit größeren Einfluß auf das Platonbild der Folgezeit, und zwar bis zum heutigen Tag, gehabt als die Arbeit der Philologen und Philosophiehistoriker vor oder nach ihm.

Überschwengliche Urteile über seine Leistung wurden schon kurz nach dem Erscheinen des ersten Bandes (1804) gefällt und mehrten sich um die Mitte des 19. Jh. Seitdem gilt es als ausgemacht, daß Schleiermacher in der Platondeutung Epoche gemacht hat.

$\mathrm{Da}$ das nichts Geringes ist, dürfte evident sein. Platon als der sprachlich und künstlerisch genialste Prosaautor der Antike (einer Epoche, die zu Schleiermachers Zeit noch als verbindlich, als klassisch galt), der zugleich inhaltlich von allen antiken Autoren die wichtigste Botschaft zu vermitteln hatte, zog von jeher eine Unzahl von Interpreten an. In dieser Atmosphäre der steten internationalen Bemühung um Platon nach langer Zeit der Gleichförmigkeit eine neue Zugangsart eröffnet zu haben, die noch dazu für fast zwei Jahrhunderte wegweisend bleiben sollte, ist eine Leistung, die für sich genommen schon ausgereicht hätte, um einen Generationen überdauernden Ruhm zu begründen. Für Schleiermacher war das nur eine von mehreren überragenden Leistungen.

Hier einige Beispiele für den hohen Ton, in dem man schon bald von ihm redete. August Boeckh, der damals noch junge, später sich als immens vielseitig und scharfsichtig erweisende Philologe, schrieb 1808 in Antizipation der Vollendung von Schleiermachers Übersetzung: „einen ächten Platon wird unsere Nation vollständig aufzuweisen haben, wie keine ihn hat, noch jemals haben wird. Lasset uns stolz darauf sein für uns, wenn auch die Fremden darauf nicht achten sollten: denn welche Nation vermöchte wohl, wie wir, den Hellenischen Weisen zu verstehen [1]?" Friedrich Ueberweg tadelte 1861 den Philologen Karl Friedrich Hermann, der neben hochtönendem Lob auch ziemlich strenge, aber sachhaltige Kritik an Schleiermacher geäußert hatte [2], folgendermaßen: ,ja, mit dürren Worten wirft Hermann seinem großen Vorgänger in der Platonischen Forschung nicht etwa nur „Fehlgriffe", sondern auch „Trugschlüsse und Verdrehungen" vor. ...Dies also ist das Bild, das wir uns von Schleiermacher's Persönlichkeit entwerfen sollen? Müssen wir so den Mann verurtheilen sehen, den wir als eine Zierde unserer Nation zu verehren gewohnt waren [3] ...?" Und Heinrich von Stein meinte 1862, Schleiermacher habe ,so gut wie zuerst den Schlüssel zur vollen Erkenntniss des ganzen Plato gefunden", er erreiche „ein so vollständiges Wiedereintreten in die platonischen Voraussetzungen, wie wir es - abgesehen von ... Schelling - nicht zum zweiten Male in der neuesten Philosophie gefunden haben", und erklärte rundheraus ,jenen Grundgedanken von Schleiermacher für das Epoche machendste Ereignis, welches in dem Verständniss der platonischen Schriften eingetreten ist, seit diese zuerst von ihrem Urheber aus der Hand gegeben worden sind [4]." 
war es möglich, daß der wahre Schlüssel zu Platon 2150 Jahre verborgen blieb, bis Schleiermacher ihn schließlich fand?

\section{II}

Doch bevor ich mich diesen Fragen zuwende, will ich kurz angeben, worin Schleiermachers Platonbild besteht [5]].

Das Besondere dieses Platonbildes ist, daß hier alles aus einem Grundgedanken folgt. Schleiermachers Forderung lautet, „Plato als philosophischen Künstler zu begreifen [] ] ". Es geht nicht darum, seine Philosophie zu begreifen und dann zusätzlich zu sehen, daß er ein überragender Schriftsteller ist. Es geht um die Unzertrennbarkeit von Inhalt und Form, genauer darum, daß die platonische Form notwendig aus einer hermeneutischen Einsicht folgt, nämlich der, daß das Erfassen eines fremden Gedankens aus einer Schrift eine Leistung der Selbsttätigkeit der Seele des Rezipienten sein muß, daß es also auf die eigene Ideenerzeugung ankommt. Der platonische Dialog ist nach Schleiermacher in allem so eingerichtet, daß er diese eigene Ideenerzeugung des Lesers hervorruft.

Schleiermachers Theorie des platonischen Dialogs gibt präzise Antworten auf die Frage nach dem Autor, dem Werk, der Absicht des Autors, der Wirkung beim Leser und nach den Mitteln, mit denen diese Wirkung erzielt wird.

1. Der Autor. (a) Er ist Philosoph und Künstler, und zwar beides in einem. (b) Er praktiziert ein „,esoterisches Handeln", bestehend im ,unmittelbaren Lehren", bei dem Platon „seine Gedanken rein und vollständig aussprechen" konnte (Einleitung 17), und ein „,exoterisches (Handeln)", bestehend im Schreiben (ib.)

2. Das Werk. (a) Es ist mit ,großer Absichtlichkeit" geschrieben, d.h. reflektiert angelegt und bewußt durchgeführt (Einl. 8), (b) es ist weder systematisch noch fragmentarisch, was die üblichen Darstellungsformen sind (Einl. 9), sondern hat eine eigene Form; ( die Dialogform ist für das platonische Werk notwendig, (d) „Form und Inhalt (sind) unzertrennlich" (Einl. 14); (e) im ganzen platonischen Werk gibt es einen ,natürlichen Zusammenhang", der zugleich die philosophische, didaktische und chronologische Abfolge der Dialoge anzeigt; (f) schließlich ist Schleiermacher der Ansicht, daß die Nachrichten über Platons ungeschriebene Lehrmeinungen, seine agrapha dogmata wie $\square$ sie Aristoteles einmal nennt, nichts enthalten, was über die Dialoge hinausginge (Einl. 13) - anders gesagt: das geschriebene Werk Platons enthält seine Philosophie vollständig.

0 3. Die Absicht des Autors ging nach Schleiermacher nicht nur darauf, (a) ,seinen eignen Sinn Andern lebendig darzulegen", (b) „sondern eben dadurch auch den ihrigen lebendig aufzuregen und zu erheben" (Einl. 14), so daß der Leser ,zur eignen inneren Erzeugung des beabsichtigten Gedankens" gebracht wird (Einl. 16). Zur Erreichung dieser doppelten Absicht müsse Platon $($ ) „gesucht haben, auch die schriftliche Belehrung jener besseren [sc. der mündlichen] so ähnlich zu machen als möglich" (Einl. 15); auf diesem Wege, glaubt Schleiermacher, habe Platon (d) „doch auch den noch nicht wissenden Leser ... zum Wissen bringen" wollen (Einl. 16) - wobei die Formulierung „,doch auch" verrät, daß Schleiermacher sich bewußt ist, daß es mit dieser für uns schlichtweg evidenten Zielsetzung angesichts des klaren Textes Platons so seine Schwierigkeiten hat.

1. Die Wirkung auf den Leser denkt sich Schleiermacher so, (a) daß es „dem Platon fast mit Jedem gelingt", (a) „entweder das zu erreichen, was er wünscht, oder" (b) „,wenigstens das zu vermeiden, was er fürchtet" (Einl. 16). 'Was er wünscht' ist, wie wir eben sahen (3 a-b), die eigenständige Erzeugung seiner Gedanken in der Seele des Lesers, 'was er fürchtet' ist, daß der Leser meint, etwas verstanden zu haben wenn das gar nicht der Fall ist. Wenn nun die eigene Ideenerzeugung, die aber ganz im Sinne des Autors sein muß, beim Leser erreicht ist, so hat sich dieser ,zu einem wahren Hörer des Inneren" erhoben (Einl. 17), im anderen Falle aber nicht, der Leser bleibt also vom Inneren ausgeschlossen, er bleibt 'draußen' (gr. exô bzw.exôteros). Diese beiden „Beschaffenheiten des Lesers" sind für Schleiermacher ,die einzige Bedeutung, in welcher man hier von einem esoterischen und exoterischen reden könnte" (Einl. 16). Esoterik ist also ein hermeneutisches Ereignis in der Seele des Lesers, nämlich die vom Text selbst gelenkte adäquate Erfassung des ,eigenen Sinns" des Autors.

0. Die literarischen Mittel, mit denen Platon die gesuchte Wirkung erzielen will, nennt Schleiermacher „Künste" (wir würden heute sagen 'Kunstmittel'). An zwei Stellen seiner „Einleitung” zählt er diese 'Künste' auf (S. 16, 30), ohne das Verhältnis der beiden Listen zueiander zu erörtern. Wenn ich richtig sehe, kommen wir zusammen auf neun einzeln benannte schleiermacherischplatonische 'Künste', die hier aufzulisten nicht müßig ist, ist doch die sogenannte indirekte Mitteilung, die sich dieser Mittel bedient, genau das, womit Schleiermacher am stärksten auf die Folgezeit gewirkt hat (abgesehen von seinem Mißverständnis des Begriffs „Esoterik"). Wir finden also bei Platon

1. das indirecte Anfangen mit etwas einzelnem,

2. eine dem Anschein nach oft willkürliche Fortschreitung, welche aber doch immer absichtsvoll und künstlich ist

3. das dialektische Verkehr mit Begriffen, worunter jedoch die Hinweisung auf das Ganze und auf die ursprünglichen Ideen immer fortgeht,

4. ein öfteres Wiederanfangen der Untersuchung von einem anderen Punkte aus, ohne Zusammenführung der verschiedenen Ansätze; ferner wird bei Platon

5. oft auf ganz fremdscheinende zufällige Art manche Andeutung hingeworfen,

6. es wird aus Widersprüchen ein Räthsel geflochten, zu welchem der beabsichtigte Gedanke die einzig mögliche Lösung ist, 7. es wird ein größeres Ziel unter einem kleineren verborgen, „oder die eigentliche Untersuchung wird mit einer andern, nicht wie mit einem Schleier, sondern wie mit einer angewachsenen Haut überkleidet" (Einl. 16)

8. „wo es auf die Darstellung eines Ganzen ankommt, da wird dieses nur durch unzusammenhängende Striche angedeutet", die der Leser aber leicht ergänzen könne (ib.). Als letzten Punkt erwähne ich den, der bei Schleiermacher als erster erscheint, nämlich 
9. "daß das Ende der Untersuchung nicht geradezu ausgesprochen und wörtlich niedergelegt" wird.

$\mathrm{Da}$ diese klaren Antworten unter fünf Aspekten, und besonders die neun Punkte unter dem fünften Aspekt der Kunstmittel, zusammengenommen eine voll entwickelte Theorie des platonischen Dialogs als philosophischer Kunstform ausmachen, ist evident. Bestritten wurde es gleichwohl (darüber gleich mehr).

III

Woher kommt Schleiermachers Theorie des Dialogs? Aus Platons Kritik des Mediums der Schrift am Ende des Dialogs Phaidros, einem sieben Druckseiten umfassenden Textstück (274 b - 278 e), aus dem Schleiermacher von (mindestens) fünf Grundgedanken [7] den ersten herausnimmt, nämlich daß die Schrift keineswegs ein Mittel zur Stärkung des Gedächtnisses ist, wie man meinen könnte, sondern nur das Ins-Gedächtnis-Zurückrufen erleichtert, das Gedächtnis selbst aber schädigt, weil die Seele das Wissen nicht mehr selbsttätig aus sich hervorholt, sondern mittels ihr äußerlicher Zeichen. Durch vieles Lesen ohne Belehrung (aneu didachês 275 a7) entstehen nur menschlich schwierige Vielwisser, statt Weisen nur eingebildete Weise (doxosophoi gegonote anti sophôn 275 b2).

Es ist nun Schleiermachers feste Überzeugung, daß Platon mit seiner Nachahmung des mündlichen Dialogs in der Schrift die Vorstellung verband, der geschriebene Dialog könne letztlich dasselbe leisten wie der mündliche, nämlich echte Belehrung durch Erregung der eigenen Ideenerzeugung beim Leser.

Die letzte Quelle der Theorie ist also eindeutig bestimmbar auch für den heutigen Leser. Weniger bekannt ist, daß Schleiermacher den Rekurs auf den Phaidros als Ausgangspunkt nicht ganz selbsttätig und als erster vornahm. In der damals noch ganz frischen „Geschichte der Philosophie" von Wilhelm Gottlieb Tennemann war zu lesen, daß Platon „,seine Schüler zum Selbstdenken gewöhnen" wollte [8] und ihnen daher in den Schriften Probleme vorlegte (daß die Dialoge Aufgaben für die Schüler enthalten, war auch die Auffassung von A. Boeckh, und in unserer Zeit etwa von R. Merkelbach [9]). Tennemann referierte die ganze platonische Schriftkritik (und nicht nur den von Schleiermacher hervorgehobenen ersten Grundgedanken) auf sieben Seiten (loc. cit. 207-14), in dem etwas früheren Werk „System der Platonischen Philosophie" hatte er den umfangreichen Text auch wörtlich abgedruckt und interpretiert [10]. Hier war zu lesen, daß „die innere und äußere Einrichtung" der platonischen Schriften vollkommen mit dem Phaidros übereinstimme, daß sich Platon also bei Abfassung seiner Schriften an „seine eigenen Grundsätze" gehalten habe. Das klingt ganz wie Schleiermacher, nur mit dem Unterschied, daß Tennemann eine esoterische Auslegung des Phaidros vertritt: Platon hatte „eine gedoppelte Philosophie, eine äußere und innere und geheime (loc. cit. 137)". Schleiermacher hingegen ließ die späteren Teile der Schriftkritik, die Tennemann zu dieser Ansicht führten, beiseite.

Neben dem Ausgang vom Phaidros ist charakteristisch vor allem die Forderung, daß in den Dialogen ein natürlicher Zusammenhang wirksam und auffindbar sein müsse - ein Gedanke, den Schleiermacher seinem Freund Friedrich Schlegel verdankte. Dieser hatte 1799 „den großen Coup" oder auch die „göttliche Idee" - so drückt sich Schleiermacher in einem Brief aus [11] - in ihm angeregt, zusammen eine Platonübersetzung zu machen. Aus dem typisch frühromantischen Plan eines intensiven 'Symphilosophierens' [12] wurde zwar nichts, der unstete Schlegel entzog sich bald dem von ihm selbst angeregten Projekt, allerdings nicht ohne es um die folgenreiche Idee einer ,historischen Ordnung" der Dialoge zu bereichern. In einem Brief an Schleiermacher vom 10. März 1800 redet er von einem „Stufengang..., als schlössen sich mehr Gespräche an einander als man gewöhnlich annimmt", und von einer „,instructiven Suite..., die die Uebersicht des Ganzen nicht wenig aufhellen würde" [13]. Schlegel wollte also den systematischen Zusammenhang von Platons Denken erfassen, sein Projekt „zielte auf ein ganzheitliches Verständnis des Platonischen Geistes", die Übersetzung war als „systematische und genetische Nachkonstruktion eines Ganzen" gedacht. [14] Diesen Gedanken hat dann Schleiermacher in „eigener Ideenerzeugung" nach der Vorgabe seines Freundes getreulich und für die ersten Leser überzeugend durchgeführt.

Dieses aus zwei so ungleichen Quellen - Tennemann und Schlegel - gespeiste neue Platonbild fand also weithin Zustimmung und Bewunderung. Ansprechend, ja zwingend war für den romantischen Zeitgeist und für die Generationen danach die Vorstellung der organischen Einheit, der „natürlichen Folge" (Einleitung 17). Alles ergibt sich hier scheinbar zwanglos aus einer einzigen kritischen Intuition Platons, von der dialogischen Gestaltung des einzelnen Abschnitts über die Anlage des betreffenden Dialogs als Ganzes bis zum übergreifenden Zusammenhang, der das platonische Oeuvre zusammenhält.

\section{IV}

Die Beziehung dieses Platonbildes zu Schleiermachers Hermeneutik ist deutlich. Auch dort geht es stets um die Möglichkeit des eigenständigen Nachvollzugs des fremden Gedankens. „Jeder Akt des Verstehens (ist) die Umkehrung eines Aktes des Redens", heißt es in der „Hermeneutik" [15]. In Schleiermachers Verständnis ist nun die platonische indirekte Mitteilungsform darauf angelegt, diese Umkehrung, die ein Zurückschreiten von der fixierten Rede zur ,inneren Rede", d.h. zum lebendigen Gedanken, ist, zu erleichtern. „Jede Rede (ist) immer nur zu verstehen aus dem ganzen Leben" (Hermeneutik 78) - ebenso ist bei Platon ,jeder Saz nur an seinem Orte und in den Verbindungen und Begränzungen, wie ihn Platon aufgestellt hat, recht zu verstehen" (Einleitung 14), und die sinnstiftenden Verbindungen ihrerseits sind nur im Kontext der „natürlichen Folge" des Ganzen zu begreifen. „Den Schwierigkeiten im Nachkonstruieren der Rede und des Gedankengangs vorzubeugen, ist die Aufgabe der Hermeneutik" (Hermeneutik 84) - die implizite Hermeneutik der platonischen Form ist für Schleiermacher die ideale künstlerische Verwirklichung dieser Forderung. „Die strengere Praxis (sc. der Hermeneutik) geht davon aus, daß sich das Mißverstehen von selbst ergibt und das Verstehen auf jedem 
Punkt muß gewollt und gesucht werden" (Hermeneutik 92) - der platonische Dialog versieht selbst diese Aufgabe der hermeneutischen Praxis, insofern der Gesprächspartner nach jeder Frage des Gesprächsführers das Aufkommen oder Ausbleiben von Verständnis signalisieren muß. Platonhermeneutik und allgemeine Hermeneutik Schleiermachers sind also aus einem Guß, und das ließe sich noch weiter im Detail ausführen.

Doch an einem Punkt wird man stutzig: wie kann ein Hermeneutiker, der klar sieht, „daß sich das Mißverstehen von selbst ergibt", zugleich der Ansicht sein, daß es Platon durch Mittel wie 'unzusammenhängende Striche', im Text ungelöste Rätsel, bloße Andeutungen und absichtliche Auslassungen gelingt, ,fast Jeden" dazu zu bringen, seinen Gedanken genuin nachzuvollziehen oder sich wenigstens nicht der Illusion hinzugeben, alles verstanden zu haben? Wenn das Mißverstehen sowieso überall lauert, so werden ihm durch die „Künste" der indirekten Mitteilungsweise nachgerade Tür und Tor geöffnet. Wieso sollen gerade diese Formen der Unbestimmtheit und Offenheit in der Seele ein saphes kai bebaionetwas 'Klares und Beständiges' (Phaidros 275c 6, 277d $\square$, 8-9, vgl. 278a 4-5) entstehen lassen? Nach Platon kann das die Schrift grundsätzlich nicht, nach Schleiermacher kann es gerade der seiner Explizitheit (gr. saphêneia) kunstvoll und systematisch beraubte schriftliche Dialog. Darin liegt ein frommer Optimismus, der mit dem Satz von der Ubiquität des Mißverstehens nicht gut zu vereinen ist.

Hier, glaube ich, liegt der eigentliche Schwachpunkt der Schleiermacherschen Dialogtheorie in hermeneutischer Hinsicht. (In anderen Hinsichten ist anderes geltend zu machen.)

Die überwältigende Zustimmung zu Schleiermacher betraf denn auch mehr die gedankenstimulierende und gedankenlenkende Funktion der Dialogform im allgemeinen als die Ausdehnung dieser Idee auf den Gesamtzusammenhang des platonischen Oeuvres. Dieser wurde bald anders bestimmt, von jedem Interpreten verschieden. Prinzipielle Zweifel an der philosophischen Notwendigkeit der Dialogform, an ihrer absoluten Unentbehrlichkeit für Platon sowie an Schleiermachers Fehleinschätzung der agrapha dogmata wurden hingegen seltengeäußert, so etwa von K. F. Hermann 1839, der aber bald von Friedrich Ueberweg zurechtgewiesen wurde [16]. Ab der Mitte des 19. Jh.s war Schleiermachers Dialogtheorie anerkannt als die unersetzbare, alle früheren Zugangsarten weit hinter sich lassende Grundlage des Platonverständnisses. Das blieb im wesentlichen so bis 1959, dem Jahr von H. J. Krämers Kritik [17], und ist bis heute gültig für die, die der Auseinandersetzung mit Krämers Ansatz aus dem Weg gehen. Durch die Emigranten der 30-er Jahre ist die Wirkung der Schleiermacherschen Dialogauffassung in der angelsächsischen Welt noch verstärkt worden und bestimmt die Zugangsart der dortigen Sekundärliteratur nicht weniger als die der hiesigen.

\section{V}

Nun gab es aber in neuerer Zeit drei Versuche, Schleiermachers Bedeutung einzuschränken:

Peter M. Steiner erklärte, Schleiermacher habe gar keine Theorie des platonischen Dialogs gehabt.

Eugène Napoléon Tigerstedt bestritt, daß Schleiermacher Epoche machte, d.h. einen entscheidenden Wendepunkt in der Geschichte der Platondeutung darstellt, und

Dorothea Frede behauptete, bei den Angelsachsen sei Schleiermacher kaum zur Kenntnis genommen worden.

Wäre auch nur eine dieser Positionen richtig, müßten wir unser Schleiermacherbild unverzüglich redimensionieren. Wären alle drei zutreffend, so würde das den großen Theologen jedenfalls auf dem Gebiet der Platonforschung zu einer eher peripheren Gestalt herabdrücken, die die Deutschen aus nationalistischer Begeisterung für diese „Zierde unserer Nation" - so hatte sich Friedrich Ueberweg ausgedrückt - zu einem Giganten aufgeblasen hätten.

Nun, es läßt sich zeigen, daß die erwähnten drei Positionen schlicht falsch sind.

Der Münchener Interpret Peter M. Steiner betrachtet die Auffassung, Schleiermacher sei „regelrecht" der Urheber „,einer neuen Theorie" als Unterstellung seiner Kritiker [18]. Leider verzichtet Steiner aber auf ein detailliertes Referat, erst recht auf eine geduldige Analyse von Schleiermachers „Einleitung" von 1804. Ich kann hier nicht nachholen, was Steiner sich selbst und seinem Leser vorenthalten hat. Es genügt aber, sich die Punkte, die ich zu Beginn dieser Vorlesung zusammengetragen habe [19], vor Augen zu halten, um zu sehen, daß Steiner Schleiermacher stark unterschätzt. Den präzisen Antworten, die Schleiermacher unter fünf klar unterscheidbaren Aspekten auf die Frage nach dem platonischen Dialog gibt, kann ein billig Urteilender den Status einer voll entfalteten, höchst reflektierten Theorie nicht absprechen.

Der schwedische Forscher Tigerstedt bestritt die übliche Einschätzung Schleiermachers als Wendepunkt in der Geschichte der Platonexegese unter der Annahme, der Ruhm Schleiermachers sei, das alte, neuplatonische Paradigma der Platondeutung zu Fall gebracht zu haben [20]. In einer großen Fleißarbeit brachte er hunderte von Belegen dafür bei, daß die neuplatonische Exegese, die Marsilio Ficino Ende des 15. Jh. wieder belebt hatte, schon hundert Jahre später, beginnend mit Johannes Serranus, abzubröckeln begann und lange vor Schleiermacher tot war. Was Tigerstedt bei allem Fleiß nicht beigebracht hat, ist auch nur ein einziger Beleg dafür, daß jemand die Dialoge vor 1804 für autark im Sinne Schleiermachers erklärt hätte, d.h. als unvollständig zwar hinsichtlich der expliziten Ausformulierung der Ergebnisse, aber doch vom Autor dazu bestimmt, seine vollständige Philosophie dem „,wahren Hörer des Inneren", also dem esoterischen Leser und nur ihm, zwischen den Zeilen sicher und verlässlich zu vermitteln. Es war aber diese Vorstellung, mit der Schleiermacher Epoche machte. 
Die Hamburger Philosophin Dorothea Frede, die 20 Jahre in den Vereinigten Staaten gelehrt hat, vertrat die Ansicht, im angelsächsischen Raum habe man „Schleiermachers Auffassung allenfalls zur Kenntnis genommen" [21], und das soll wohl heißen: Einfluß hat Schleiermacher nicht gehabt ( - und vielleicht ist noch etwas Weitergehendes gemeint, nämlich: was bei der Leitkultur des 20. Jh. keinen Eindruck gemacht habe, könne ja wohl nicht viel wert sein). Ich fürchte, Frau Frede steht allzu sehr im Bann der amerikanischen Unkenntnis der eigenen Wurzeln. Es läßt sich durchaus zeigen, daß Schleiermacher nicht nur von Anfang an rezipiert wurde - eine englische Übersetzung seiner Einleitungen zu Platon durch W. Dobson erschien bereits 1836 in London, zwei Jahre nach Schleiermachers Tod -, sondern auch intensiv gewirkt hat [22]. Die Tatsache, daß manche amerikanische Kollegen (und solche, die in den Staaten unterrichtet haben) nicht wissen, wo die Wurzeln ihrer Platonhermeneutik liegen, ändert nichts daran, daß die gegenwärtige angelsächsische Platondeutung fest auf dem von Schleiermacher geebneten Boden steht.

Es ist also nichts mit den neuesten Einschränkungen der Bedeutung Schleiermachers. Man fragt sich: wie kamen sie überhaupt zustande? Und hier zeigt sich dem erstaunten Blick eine Gemeinsamkeit: Steiner, Tigerstedt und Frede sind alle drei erbitterte AntiEsoteriker. Alle drei schreiben mit spürbarem Engagement an gegen die, die eine mündliche Prinzipientheorie Platons, die in mehreren Punkten klar über die Dialoge hinausging, für eine historische Tatsache halten. Um diese Gegner zu treffen, sind sie bereit, Schleiermacher entweder zur Randfigur zu machen oder ihm einen seiner Ruhmestitel, seine geniale Theorie des platonischen Dialogs, zu nehmen.

\section{VI}

Wir kommen also nicht darum herum, doch noch über Schleiermachers Stellung zur Frage einer esoterischen Philosophie Platons ausführlich zu sprechen (bisher begnügten wir uns mit Andeutungen). Die Frage ist offenbar bis heute ein wunder Punkt in der Diskussion um Platon.

Nur vier Jahre nach dem Erscheinen der epochemachenden Einleitung schrieb A. Boeckh, Schleiermacher sei in der Frage der platonischen Esoterik ein Proteus, den man nicht recht fassen könne [23]. Boeckh hatte vollkommen Recht: in unmittelbarem Anschluß an seine neue Deutung von Esoterik als eines vom Text selbst induzierten innerseelischen Ereignisses im Leser und an seine Erklärung, das dies „die einzige Bedeutung [sei], in welcher man hier von einem esoterischen und exoterischen reden könnte", erkennt Schleiermacher auch jene andere Art von Esoterik rückhaltlos an, die darin bestand, daß Platon seine Gedanken ,rein und vollständig" nur im mündlichen Unterricht aussprach, ,wenn er erst hinlänglich gewiss war, die Hörer seien ihm nach Wunsch gefolgt" (Einleitung 16f.). Was soll nun gelten? Gibt es nur eine einzige Bedeutung von esoterisch, oder doch zwei? In der allerverbindlichsten Form, ohne jede Schärfe der Polemik, stellt Boeckh fest, daß Schleiermacher weder die einschlägigen „Äußerungen Platons" noch die „,weit verbreitete Überlieferung aus dem Alterthum" über platonische Esoterik genauer untersucht hat. Ihm, Boeckh, scheint platonische Esoterik wahrscheinlich schon wegen Platons ,unverkennbarer Hinneigung zum Pythagoreismus" (6). Platon schrieb gewiß ,auch für das grössere Publicum", vor allem aber für seine Schüler in der Akademie im Sinne einer Aufgabenstellung. Das Schriftliche ist nach Boeckh nicht bis zur höchsten Spitze hinaufgeführt, das Mündliche hingegen hat diese fehlende Spitze, den „Schlußstein", aufgesetzt. Und dann sagt Boeckh etwas überaus Wichtiges, das viele Platoniker bis heute nicht bedacht haben: In den Schriften selbst „,müßte sich Platon auf die wunderlichste Weise geziert haben, wenn er nichts Esoterisches gehabt hätte" (7). Boeckh verweist auf den 7. Brief sowie auf jene Stelle in Platons Politeia, wo Sokrates erklärt, daß seine Ansicht vom Wesen (vom ti estin) des Guten hier nicht zur Sprache kommen soll, vielmehr statt dessen nur das Gleichnis von der Sonne als Analogon der Idee des Guten (506 de), ,,andeutend zugleich, daß er wohl noch mehr zu sagen hätte: wo sollte er dies getan haben, als in seinen Vorträgen?" Boeckh beharrt nun darauf, daß dieser Verweis des Hauptwerks Platons auf ausstehendes Mehrwissen über das Gute etwas zu tun hat mit den antiken Berichten über die mündliche Philosophie Platons, in der die philosophische Bestimmung des Guten das zentrale Thema war. Anders gesagt: wer den Wortlaut des geschriebenen Werks gebührend beachtet, kann unmöglich das inhaltliche Plus des Mündlichen bei Platon leugnen. Das aber hatte Schleiermacher explizit getan.

Was Boeckh hier unternahm, war ein unendlich behutsamer Versuch, die Diskussion um den mündlichen Platon wieder auf den Stand zu heben, den sie bei Dieterich Tiedemann und Wilhelm Gottlob Tennemann in den 90-er Jahren des 18. Jh. gehabt hatte. Daß Schleiermacher hinter diesen Stand zurückfiel, sollte man heute nicht leugnen [24] Zu bestreiten, daß die antiken Berichte über Platons agrapha dogmata ein substantielles dogmatisches Mehr gegenüber dem Dialogwerk aufweisen, war 1804 nicht vernünftiger oder entschuldbarer als es heute wäre. Tiedemann und Tennemann hatten dieses Mehr zwar nicht vollständig aus den Quellen aufgespürt und interpretiert, aber doch so weit sichtbar gemacht, daß nach ihren Bemühungen ein Ignorieren oder gar Leugnen befremdlich bleiben muß.

\section{VII}

Das am Ende des 18. Jh. erreichte Platonbild umfaßte u. a. folgende Punkte [25]:

Platon wollte nicht das Ganze seines Systems in den Dialogen mitteilen; die Dialoge sind ihrer Natur nach exoterisch;

国 die Form der mündlichen Lehre war eine andere als die der schriftlichen Darlegungen: frei vom Zwang zur dialogischen Einkleidung, ohne dichterische Verzierungen wie Mythen und Gleichnisse;

platonische Belege für eine esoterische Lehre sind die Schriftkritik im Phaidros, der 7. Brief sowie die Aussparungsstellen, d.h. jene typisch wiederkehrenden Stellen, an denen der Gesprächsführer klar zu erkennen gibt, „daß er noch mehr zu sagen hätte" (wie 
Boeckh es formulierte);

die Motive für Platons Zurückhaltung in der Schrift liegen in seiner geringen Einschätzung der Möglichkeiten dieses Mediums in der philosophischen Gedankenerweckung, in der Überzeugung, daß vieles zu schwer, zu voraussetzungsreich ist, um einfach so herumgereicht zu werden, und schließlich in seiner Verantwortung für die Sache und seiner Reverenz für die göttlichen Dinge, auf die sich sein Denken richtete;

als Quellen der mündlichen Lehre betrachtete man die von Aristoteles zitierten Werke Dihaireseis und Peri philosophias, die man irrtümlich für platonische esoterische Schriften hielt, wie auch der Titel agrapha dogmata als Bezeichnung einer solchen Schrift galt;

die aristotelischen und sonstigen Berichte galten als zwar lückenhafte und teilweise unklare, im wesentlichen aber verläßliche Zeugnisse der mündlichen Philosophie Platons;

国 diese Philosophie wurde verstanden als eine Allgemeine Ontologie (Tiedemann), oder als eine Art Transzendentalphilosophie (Tennemann); Dialoge und mündliche Prinzipienlehre repräsentieren - wie aus Aristoteles Met. A 6 gefolgert werden kann - zwei verschiedene Stufen der Zurückführung der Dinge auf ihre Prinzipien: die Dialoge verfolgen diesen Weg nur bis zu den Ideen, die mündliche Philosophie bis zu den ersten Prinzipien, der Idee des Guten oder Einen und dem negativen Prinzip der Unbestimmten Zweiheit [26].

Hiervon bleibt bei Schleiermacher scheinbar viel erhalten. Daß Platon ein System hatte, sagt auch er, ebenso, daß die Dialoge unvollständig sind und daß deren Abfassung nur das exoterische Handeln Platons darstellte; ferner, daß Platons Gedanken ,rein und vollständig" und in wissenschaftlicher Durchführung in die mündliche Lehre gehörten. Gewichtiger ist aber, was wegbleibt. Die Aussagekraft der Zeugnisse über den Inhalt der agrapha dogmata wird, wie wir sahen, pauschal und ohne Prüfung bestritten. Die platonischen Stellen, die ein esoterisches Verhalten des Philosophen nahe legen bzw. behaupten, bleiben in der Einleitung unerwähnt, vier Fünftel der Schriftkritik finden keine Berücksichtigung. Von den Motiven, die einen Denker zur Zurückhaltung in der Schrift veranlassen können, erwähnt Schleiermacher nur das politische und das didaktische Motiv, d.h. nur die Angst vor dem Konflikt mit der polytheistischen Volksreligion, was nach seiner Meinung Esoterik „zu einer kindischen Veranstaltung" (Einleitung 12) machen würde, sowie die mangelnde Vorbereitung und Schulung der Leser. Schwer verständlich, wie der Ethiker und Theologe Schleiermacher das ethische und das religiöse Motiv übergehen konnte, d.h. einmal die Forderung Platons, daß zur Dialektik im besten Staat nur zugelassen werde, wer in jahrelanger Charakterschulung alle Tests überstanden hat (Politeia 535 a ff. [vgl. 485 a 487 a, u.ö.]) (wobei unbestritten ist, daß Bücher die ethische Formierung nicht leisten können), und zweitens die Tatsache, daß Platon die Ideen als den Bereich des Göttlichen ansah (Politeia 550 c [u.o.]), zu dem er mit religiöser Scheu aufblickte, weswegen es ihm auch nicht gleichgültig war, in wessen Hände seine Bemühungen um diesen Bereich gerieten und wie sie aufgenommen wurden (Politeia 536 c, 539 b-d; Siebter Brief 341 d-e, 344 c-e). Dieses religiöse Motiv, das für Platon von den vier Motiven wohl das stärkste war, hatte Tennemann, den Quellen folgend, klar benannt [27], doch der Theologe, der das religiöse Gefühl für seine Zeit neu bestimmt hat, Schleiermacher, ließ es gänzlich beiseite - wie gesagt, ein Rätsel. Wegbleiben mußte notwendig auch die Vorstellung von zwei qualitativ verschiedenen Stufen der Annäherung an die Prinzipien im Schriftwerk und in der mündlichen Lehre. Denn wenn das geschriebene Werk den Leser sicher von Dialog zu Dialog führen kann, auch wenn das Ende nicht ausgesprochen sondern vom Rezipienten zu ergänzen ist, so kann es wohl auch die nicht ausgesprochenen letzten Einsichten, die „rein und vollständig" in der mündlichen Lehre vorkamen, zwischen den Zeilen vermitteln.

\section{VIII}

Man könnte nun einwenden: wenn Schleiermacher Platon sowohl die textimmanente oder hermeneutische Esoterik zuschreibt, also die gezielte Erzeugung von Verständnis durch den Text selbst bei bestimmten privilegierten Lesern, den ,wahren Hörern des Inneren", als auch die Esoterik im üblichen Sinn, wonach eine inhaltlich über die Dialoge hinausgehende mündliche Lehre in der Akademie eine historische Realität war, so ist doch alles in Ordnung. Konrad Gaiser hat in seinen Neapler Vorlesungen „Platone come scrittore filosofico" 1984 überzeugend dargelegt, daß die von ihm vertretene esoterische Position die textimmanente Esoterik widerspruchsfrei integrieren kann: Platon konnte, sagt Gaiser, sowohl bestimmte Lehrmeinungen von der Verschriftlichung überhaupt ausschließen als auch bestimmte andere Einsichten mittels der indirekten Mitteilungsart zwischen den Zeilen nur den Verständigen vermitteln. Die beiden Entscheidungen des Autors kommen nirgends in Konflikt miteinander, ergänzen sich vielmehr sinnvoll. Und hier zeigt sich die Überlegenheit von Gaisers hermeneutischem Standpunkt: er vertritt die umfassendere und vorurteilsfreiere Dialogtheorie, die weder blind ist für die von Schleiermacher aufgezeigten Vorzüge der indirekten Mitteilung noch dazu verurteilt ist, die umfangreiche historische Überlieferung zum mündlichen Platon über Bord zu werfen (im Gegensatz zu seinen anti-esoterischen Gegnern der 70-er und 80-er Jahre). Erkennt nicht auch Schleiermacher beide Formen von Esoterik an?

Ja, er tut es, aber im Fall der historischen Esoterik nur mit spürbarem Widerwillen und in Form eines bloßen Lippenbekenntnisses vermutlich weil er spürte, daß er sie argumentativ nicht erledigt hatte (was ja Boeckh schon vier Jahre darauf feststellte). Das eigentliche Ziel der schleiermacherschen Platonhermeneutik ist aber, die Esoterik der historischen Zeugnisse zu ersetzen durch die von ihm neu beschriebene und neu in den Vordergrund gerückte textimmanente Esoterik. Es war ja nach seiner Überzeugung Platons Ziel, die schriftliche Belehrung der mündlichen nach Möglichkeit anzunähern, und das müsse Platon auch gelungen sein (Einleitung 15). Die Schrift enthält alles, und sie kann alles. Dann braucht man aber die von Aristoteles, Theophrastos, Alexander von Aphrodisias und anderen bezeugte esoterische Lehre nicht mehr. Eben diese Tendenz zur Verdrängung der agrapha dogmata im 
Vertrauen auf die Allkompetenz der Schrift war das, womit Schleiermacher Epoche machte.

Hinter dieser Tendenz steht aber unausgesprochen ein Schluß, der nicht trägt. Daraus, daß ein Autor sich entschlossen hat, gewisse Inhalte nur in der Form der indirekten Mitteilung zu verschriftlichen, damit der Leser sie sich selbst erarbeite, folgt natürlich nicht, daß derselbe Autor sich entschließen müßte, oder gar sachlich gezwungen wäre, alles, was ihm philosophisch wichtig ist, in dieser Form der Schrift anzuvertrauen. Dazu bedürfte es einer zusätzlichen Entscheidung des Autors. Im Falle Platons aber ist eine solche Entscheidung nicht nur nicht bekannt, vielmehr wird die entgegengesetzte Entscheidung, nicht alles in die Schrift zu bringen, durch den Phaidros und die Aussparungsstellen nahe gelegt, durch den 7. Brief direkt bezeugt.

Was am Ende herauskommt bei Schleiermachers Platonhermeneutik, ist die Auffassung, daß der geschriebene Dialog in der Lage ist, durch subtile Lenkung des Lesers genuin philosophische Erkenntnis zu erzeugen, daß also die Schrift dazu geeignet ist, „doch auch den noch nicht wissenden Leser ... zum Wissen (zu) bringen" (vgl. Einleitung 16). Das ist das Gegenteil von dem, was Platons Schriftkritik besagt. Friedrich Nietzsche sah das: in seiner Basler Vorlesung von 1871/72 arbeitete er den Gegensatz zwischen Platon und Schleiermacher so heraus: für den letzteren ist die Schrift „das zweitbeste Mittel, den nicht Wissenden zum Wissen zu bringen", Platon dagegen sage, ,nur für den Wissenden, als Erinnerungsmittel, habe die Schrift Bedeutung". Für Nietzsche steht Schleiermachers „ganze Hypothese im Widerspruch zu der Erklärung im Phädrus und ist durch eine falsche Interpretation befürwortet" [28].

Wenn der geschriebene Dialog bei Schleiermacher insgesamt das leisten kann, was bei Platon nur die mündliche Lehre des Dialektikers leistet, dann muß der Dialog auch von den drei Mängeln der Schrift einzeln frei sein. Diese sind nach Platon: 1. die Schrift kann auf Fragen nicht antworten, 2. sie kann nicht reden oder schweigen, zu wem man reden oder schweigen sollte, und 3. sie kann sich bei Angriffen nicht selbst zu Hilfe kommen, sondern bedarf der Hilfe durch den Autor. Schleiermacher selbst hat diese drei Mängel in seiner Einleitung nicht einzeln aufgezählt, folglich mußte er auch nicht versichern, daß der platonische Dialog seiner Ansicht nach diese Mängel hinter sich zu lassen vermag. Solch eine Versicherung gab später, in expliziter Fortführung des schleiermacherschen Ansatzes, etwa Heinrich von Stein: „Die platonischen Schriften sind in unsern Augen wirklich von der Art, dass sie den ungehörigen Leser ganz abschrecken, ..., dass sie Rede und Antwort stehen auf ... Fragen und Einwendungen, dass sie ... in die Seelen geschrieben sind ..." [29]. Hier sind wir nun ganz konsequent beim plattesten Widerspruch zu Platons Schriftkritik angelangt, denn all das kann nach Platon diegraphê die Schrift ganz allgemein und ohne Ausnahme, gerade nicht, und in die Seele des Hörers geschrieben (statt aufs Papier) ist bei Platon allein „die lebendige und beseelte Rede des Wissenden" (ho tou eidotos logos zôn kai empsuchos, Phaidros 275 a8), also die mündliche Dialektik. Doch in dieser Form, mit dem direktesten Widerspruch zu den Aussagen der Schriftkritik, hat Schleiermachers Platonhermeneutik die Platonexegese des 19. und 20 Jh. in ihren Bann gezogen. Die textimmanente Esoterik hat über die von den Quellen geforderte Anerkennung des Unterschieds von Schriftwerk und mündlicher Philosophie bei Platon gesiegt. Der Preis für diesen Sieg war u. a. eine Theorie des platonischen Dialogs als eines Wunderdings, das all das leisten kann, was Platon ihm explizit absprach. [30] Die führende Platonhermeneutik des 19. und 20. Jh. war nicht unplatonisch, sie war antiplatonisch.

IX

Was ist zu tun für den Platoniker? Schleiermachers An-den-Rand-Drängen der mündlichen Philosophie Platons ist zurückzunehmen, womit schon 1908 Léon Robin, in den 20-er Jahren Julius Stenzel begonnen hatten, bevor H. J. Krämer und Konrad Gaiser seit 1959 bzw. 1963 energisch auf die Testimonien der agrapha dogmatazurückgriffen. [31] Das inhaltliche Plus dieser Überlieferung ist unbestreitbar und eine wertvolle Ergänzung und Hilfe für die Interpretation der Dialoge. Die Motive Platons für seine Zurückhaltung in der Schrift sind klar herauszuarbeiten: es gab politische, didaktische, ethische und religiöse Motive. Die Analyse der Motive zeigt, daß Esoterik nicht dasselbe ist wie Geheimhaltung. Daß die textimmanente Esoterik grundsätzlich nicht geeignet ist, die von den Testimonien bezeugte Esoterik Platons zu verdrängen, sollte endlich anerkannt werden.

Soll man also Schleiermacher über Bord werfen in Platonicis? Keineswegs. Schleiermachers Beschreibung der 'Künste' der indirekten Mitteilung hat unsere Art, Platon zu lesen, verändert, und das kann nicht aufgegeben werden. Schleiermachers Ansatz ist vielmehr fortzuführen.

Einmal ist an der Richtigkeit von Schleiermachers proleptischer Lesart der Dialoge festzuhalten. Es trifft zu, daß alles, was Platon schreibt, im Vorgriff auf weiter reichendes, tiefer begründendes Wissen geschrieben ist, das jedoch an der betreffenden Stelle nicht entfaltet, wohl aber vorausgesetzt und oft auch andeutend umschrieben wird. Schleiermacher nun nahm als Bezugspunkt solcher Prolepsis die Politeia, die er als das konstruktive Hauptwerk der letzten Phase des platonischen Schaffens betrachtete. Heute glauben wir zu wissen, daß die Politeia in die mittlere Periode gehört. Folglich kann die proleptische Bezugnahme auf das nicht ausbuchstabierte, aber im Hintergrund präsente fundierende Wissen nicht mehr einstufig konzipiert werden, wie es vor kurzem noch Charles Kahn [32] mit Rückgriff auf Schleiermachter tat, sondern notwendig zweistufig, denn die späten Dialoge sind ja nicht weniger deutlich proleptisch angelegt als die frühen. Es sind also die frühen Dialoge proleptisch vom Horizont der Politeia her geschrieben, die Politeia ihrerseits und die späten Dialoge proleptisch vom Horizont der ungeschriebenen Prinzipienlehre her.

Festzuhalten ist ferner Schleiermachers Einsicht, daß Form und Inhalt bei Platon unzertrennlich sind. Schleiermacher selbst hat diese Einsicht freilich nur hinsichtlich der Wirkungsweise der Kunstmittel der indirekten Mitteilungsform entfaltet. Diese ist weiter zu 
analysieren, wobei eher die Korrektur früherer Übertreibungen das Ziel sein sollte als das Aufspüren neuer tiefsinniger Doppeldeutigkeiten und versteckter Anspielungen. Der Gedanke der Einheit von Form und Inhalt ist über Schleiermacher hinaus auszuweiten auf die dramatische Gestaltung der Dialoge: es ist zu zeigen, daß Handlung und Figurenkonzeption immer wieder auf das Bild von esoterisch verfahrenden Gesprächsführern führen, so etwa wenn es in der Politeia niemandem gelingt, die Zurückhaltung des Sokrates hinsichtlich seiner Ansichten über Dialektik und die Idee des Guten aufzulockern, oder wenn in allen Dialogen der Gesprächsführer konzipiert ist als einer, der seinem Partner haushoch überlegen und der aktuellen Gesprächsphase meilenweit voraus ist. Wenn Platon solche Figuren auch noch sagen läßt, daß es Weiteres und Wichtigeres gibt, das indes hier und jetzt nicht zur Sprache kommen soll, so wäre er selbst als Autor eine schillernde und unglaubwürdige Gestalt, wenn diese Andeutungen einer möglichen tieferen Fundierung ungedeckte Schecks wären, ausgestellt, um sein eigenes Bild größer erscheinen zu lassen als es war.

Solche weitere Analyse der platonischen Form, die in den letzten 20 Jahren vielerorts schon betrieben wird - wenn auch für gewöhnlich ohne Rückbezug auf Platons Schriftkritik - ist zweifellos eine legitime und notwendige Fortsetzung der Bemühungen Schleiermachers. Fortsetzung verdient und verlangt auch sein Ansatz bei der Schriftkritik, von der er nur einen einzigen zentralen Gedanken entfaltete: nimmt man, auf seiner Spur weiterschreitend, die weiteren Aussagen hinzu, so erkennt man, daß Platon vom Philosophen fordert, er müsse grundsätzlich in der Lage sein, im mündlichen Gespräch seine eigene Schrift inhaltlich in den Schatten zu stellen - was doch wohl nur möglich ist, wenn er seine Schriften so anlegt, wie Platon seine angelegt hat, nämlich bewußt unvollständig hinsichtlich der letzten Begründung, die eben der mündlichen 'Hilfe für den Logos' vorbehalten bleiben muß.

So zeigt sich denn die Größe und bleibende Lebendigkeit von Schleiermachers Entwurf: um als Platoniker dem Objekt unserer hermeneutischen Bemühungen näher zu kommen, können wir nicht gegen ihn ankämpfen, vielmehr bleibt uns keine andere Wahl als Schleiermacher auf dem durch ihn selbst aufgewiesenen Weg zu überwinden.

\section{THOMAS ALEXANDER SZLEZÁK}

Footnotes

[1] A. Boeckh, Gesammelte Kleine Schriften, 7. Band: Kritiken, Leipzig 1872, 38.

[2] K. F. Hermann, Geschichte und System der Platonischen Philosophie, Heidelberg 1839, 359-368.

[3] F. Ueberweg, Untersuchungen über die Echtheit und Zeitfolge Platonischer Schriften, Wien 1861, $50 \mathrm{f}$.

[4] H. von Stein, Sieben Bücher zur Geschichte des Platonismus. 3 Bände, Göttingen 1862-1875; Zitate I 34 Anm. 1, III 374, I 33

Anm. 1.

[5] Die folgende Skizze stützt sich vor allem auf die „Einleitung" zur Gesamtübersetzung in: „Platons Werke von Friedrich Schleiermacher. Ersten Theiles erster Band. Berlin 1804". Zitiert wird nach der Seitenzählung der 3. Auflage (Berlin 1855: Einleitung S. 5-36), die auch im Nachdruck von K.Gaiser (in: Das Platonbild, Hildesheim 1969, 1-32) am Rand angegeben ist. Bequem zugänglich ist der Text auch bei P.M. Steiner (Hrsg.): Friedrich Daniel Ernst Schleiermacher, Über die Philosophie Platons, Hamburg 1996, 25-69. - Schleiermachers Einleitungen zu den einzelnen Dialogen bieten demgegenüber kaum Bemerkungen von grundsätzlicher Bedeutung, die über die Gesamt-Einleitung hinausgehen würden, während das Kapitel „Platon selbst" in Schleiermachers Vorlesung „Geschichte der Philosophie" von der spezifisch schleiermacherschen Auffassung, wie sie im 19. und 20. Jh. gewirkt hat, so gut wie nichts durchscheinen läßt. Ein (an Druckfehlern reicher) Abdruck dieses Kapitels findet sich bei P.M. Steiner (s.o.), 7-20.

[6] H. von Stein, loc. cit. III 353, nach Schleiermacher, Einleitung 7.

[7] Die wichtigsten Punkte der Schriftkritik sind: (1) Die Schrift hilft nur, schon Bekanntes in Erinnerung zu rufen, stärkt aber nicht das Gedächtnis; sie erzeugt nicht Weisheit, sondern nur den Schein derselben (Phdr. 274 c - 275 d). (2) Die Schrift hat drei Mängel: sie kann nicht antworten, sie weiß nicht, zu wem sie reden und zu wem sie schweigen soll, und sie kann sich bei Angriffen nicht selbst zu Hilfe kommen (275 de). (3) Von diesen drei Mängeln ist allein die lebendige Rede des „Wissenden", d.h. des Dialektikers, frei (276a). (4) Der Dialektiker wird mit seiner philosophischen 'Saat' so umgehen wie der kluge Bauer: er wird nicht sein gesamtes 'Saatgut' in 'Adonisgärten' (= Schriften) ausbringen (276 b - 277 a). (5) Der Dialektiker (= philosophos) kann seine Schriften durch seine mündliche Stellungnahme als 'gering' oder 'nicht fachmännisch' (phaula erweisen und im Prozeß der mündlichen 'Hilfe' für seinen Logos 'Dinge von höherem Wert' timiôtera zum Vorschein bringen (278 c-e). Als sechsten (bzw. ersten) Leitgedanken könnte man hinzufügen, daß nur der Umgang des Philosophen mit den Logoi 'gottgefällig' ist (274 a).

[8] W. G. Tennemann, Geschichte der Philosophie, Band II Leipzig 1799, 200

[9] A. Boeckh, Ges. Kleine Schriften (oben Anm. 1) VII 6; R. Merkelbach (Hrsg.), Platons Menon, hrsgg., übersetzt und nach dem Inhalt erläutert von R.M., Frankfurt/Main 1988 (bes. 5-10).

[10] W. G. Tennemann, System der Platonischen Philosophie, Band I (Leipzig 1792) 129-38.

[11] Brief an H. Herz vom 29. 4. 1799: „Schlegel schrieb mir ... von einem großen Coup den er noch vorhätte mit mir und das ist nichts geringeres als den Plato übersetzen. Ach! es ist eine göttliche Idee, ..." (Friedrich Daniel Ernst

Schleiermacher, Briefwechsel 1799 - 1800 (Briefe 553-849) hrsgg. von A. Arndt und W. Virmond, Berlin-New York 1992 [ = Kritische Gesamtausgabe, hrsgg. von H.-J. Birkner et al., Berlin-New York 1984 ff., hier: Band V 3], Brief 640, S. 101). Zwischen Schlegel und Schleiermacher scheint „,nur eine einzige ordentliche Unterredung über den Platon" stattgefunden zu haben, s. Wilhelm Dilthey, Leben Schleiermachers, Erster Band, Berlin 1870, 534. Genaue Nachweise über die Rolle, die der Plan der Platon- 
Übersetzung in Schleiermachers Briefwechsel spielt, finden sich bei H. von Stein, Sieben Bücher (s.o. Anm. 4), $343-5$ (Anm. 8 von S. 343).

[12] Vgl. Arndt (s. Anm. 14) XI.

[13] Brief 808 (Schlegel an Schleiermacher) in: F.D.E. Schleiermacher, Briefwechsel 1799 - 1800 (=KGA V 3) (s.o. Anm. 11 ), 412.

[14] Ich übernehme hier Formulierungen von Andreas Arndt, Schleiermacher und Platon, in: P. M. Steiner (Hrsg.) (oben Anm. 5),

VII-XXII, hier: XII. - Für eine geistesgeschichtlich und philosophisch vertiefte Auseinandersetzung mit Schlegels Platonbild und Philosophieverständnis sowie deren Einfluß auf Schleiermacher - Aspekte, die Arndt nicht behandelt - vgl. H. Krämer, Fichte,

Schlegel und der Infinitismus in der Platondeutung, in: Deutsche Vierteljahrsschri fürLiteraturwissenschaft und

Geistesgeschichte 62, 1988, 583-621, bes. 600-10.

[15] F.D.E. Schleiermacher, Hermeneutik und Kritik, hrsgg. und eingeleitet von Manfred Frank, Frankfurt a. M., 1999, S. 76.

[16] K.F. Hermann, loc. cit (oben Anm. 2); ders., Über Platons schriftstellerische Motive (1839), in: Gesammelte Abhandlungen, Göttingen 1849, 281-305 (Nachdruck bei K. Gaiser (Hrsg.), Das Platonbild, 1969, 33-57); F. Ueberweg, Untersuchungen ... (s.o. Anm. 3), 43-111.

[17] H.J. Krämer, Arete bei Platon und Aristoteles. Zum Wesen und zur Geschichte der platonischen Ontologie, AHAW 1959 (6), Heidelberg 1959; vgl. ders., Platone e I fondamenti della metafisica, Milano 1982, 3. Aufl. 1989, 33-149.

[18] P.M. Steiner, Zur Kontroverse um Schleiermachers Platon, in: P.M. Steiner (oben Anm. 5) XXIII-IXIV, bes XXXIV: „Szlezák unterstellt Schleiermacher regelrecht eine neue Theorie, die 'moderne Theorie des platonischen Dialogs".

[19] Eine detaillierte Auseinanadersetzung mid Schleiermachers Dialogtheorie findet sich in „Platon und die Schriftlichkeit der Philosophie", 1985, 331-75.

[20] E.N. Tigerstedt, The Decline and Fall of the Neoplatonic Interpretation of Plato, Helsinki 1974. Vgl meine Rezension in GGA 230, 1978, 33-7.

[21] D. Frede, „Die wundersame Wandelbarkeit der antiken Philosophie in der Gegenwart", in: E.-R. Schwinge (Hrsg.) Die Wissenschaften vom Altertum am Ende des 2. Jahrtausends $n$. Chr., Stuttgart 1995, 31.

[22] Einige mehr provisoriche Hinweise dazu stellte ich zusammen in: „Schleimachers 'Einleitung' zur Platon-Übersetzung von 1804. Ein Vergleich mit Tiedemann und Tennemann", Antike und Abenland 43, 1997, $61 \mathrm{f}$.

[23] Ges. K1. Schr., Band 7, 1972, 8.

[24] Zum Verhältnis der Platonhermeneutik Schleiermachers zu der seiner Vorgänger Tiedemann und Tennemann s. meinen oben Anm. 22 genannten Beitrag.

[25] Die Nachweise im einzelnen, die ich in AuA 43, 1997, 47-53 (vgl. oben Anm 22) zusammengestellt habe, möchete ich hier aus Platzgründen nicht in ihrer Gesamtheit wiederholen. Vgl. unten Anm. 26 und 27.

[26] Dieterich Tiedemann, Geist der spekulativen Philosophie II (Marburg 1791) 73; vgl Tennemann, System (oben Anm. 10) I 137.

[27] W.G. Tennemann, System der Platonischen Philosophie I (1792), 129; ders., Geschichte der Philosophie II (1799) $214 \mathrm{f}$.

[28] Nietzsche's Werke, Band XIX, Dritte Abteilung: Philologica, Band III: Unveröffentlichtes zur antiken Religion und Philosophie, hrsgg. von Otto Crusius und Wilhelm Nestle, Leipzig 1913, darin S. 235-304: „Einführung in das Studium der platonischen Dialoge", Zitate: 240.

[29] H. von Stein, loc. cit. (oben Anm. 4) I 72 f.; vgl. 70: „Der wahre Philosoph ... kann selbst solche Schriften hervorbringen, die sich allein zu vertheidigen wissen, ..."

[30] Zur gleichsam mythischen Überhöhung der wundersamen Fähigkeiten der Dialogbuches in der modernen Dialogtheorie s. „Platon und die Schriftlichkeit der Philosophie", 1985, 353-8.

[31] L. Robin, La théorie platonicienne des idées et des nombres d'après Aristote, Paris 1908; J. Stenzel, Studien zur Entwicklung der platonischen Dialektik von Sokrates zu Aristoteles, 1917 (2. Aufl. 1931); ders., Zahl und Gestalt bei Platon und Aristoteles, Leipzig/Berlin 1924 (2. Aufl. 1933); H.J. Krämer, Arete bei Platon und Aristoteles (s.o. Anm. 17); K. Gaiser, Platons ungeschriebene Lehre. Studien zur systematischen und geschichtlichen Begründung der Wissenschaften in der Platonischen Schule, Stuttgart 1963 (2. Aufl. 1968).

[32] Ch. H. Kahn, Plato and the Socratic Dialogue. The Philosophical Use of a Literary Form, Cambridge 1996 - Kahn stellt zwar gebührend heraus, daß seine 'proleptische' Lesart der Dialoge den Ideen Schleiermachers verpflichtet ist. Was hingegen nicht klar genug heraus-kommt, ist die Tatsache, daß die 'proleptische' Lesart in der deutschspragigen Platonliteratur stets intensiv gepflegt wurde, auch wenn das Wort 'proleptisch' nicht immer dafür verwendet wurde. Als Beispiel nehme man etwa W. Schulz, Das Problem der Aporie in den Tugend-dialogen Platos (in: Die Gegenwart der Griechen im neueren Denken, Festschrift für Hans-Georg Gadamer, Tübingen 1960, 261-75), oder auch meine Interpretationen der frühen und mittleren Dialoge in „Platon und die Schriftlichkeit der Philosophie" (1985), wo ich mein ganzes Unterfangen in der Schlußbemerkung (328 f.) als neu begründete Empfehlung der proleptischen Lektüre (unter Verwendung dieses Wortes) einordne. 